\title{
Impact of alcohol septal ablation on left anterior descending coronary artery blood flow in hypertrophic obstructive cardiomyopathy
}

\author{
Willem G. van Dockum · Paul Knaapen · Mark B. M. Hofman \\ Joost P. A. Kuijer · Folkert J. ten Cate $\cdot$ Jurrien M. ten Berg • \\ Aernout M. Beek · Jos W. R. Twisk $\cdot$ Albert C. van Rossum
}

Received: 5 August 2008/Accepted: 3 February 2009/Published online: 22 February 2009

(C) Springer Science+Business Media, B.V. 2009

\begin{abstract}
Objectives The aim of this study was to evaluate the effects of alcohol septal ablation (ASA) on coronary blood flow in symptomatic hypertrophic obstructive cardiomyopathy (HOCM) using cardiac MR (CMR) coronary flow measurements. Background CMR flow mapping enables quantification of coronary blood flow in a noninvasive way. Both left ventricular outflow tract (LVOT) gradient reduction and myocardial scarring after ASA are expected to influence left anterior descending (LAD) coronary
\end{abstract}

W. G. van Dockum $(\varangle) \cdot$ P. Knaapen ·

A. M. Beek - A. C. van Rossum

Department of Cardiology, ICaR-VU, VU University Medical Center, De Boelelaan 1117, P.O. Box 7057, 1081 HV Amsterdam, The Netherlands

e-mail:wg.vandockum@vumc.nl

M. B. M. Hofman · J. P. A. Kuijer

Department of Physics and Medical Technology,

ICaR-VU, VU University Medical Center,

Amsterdam, The Netherlands

F. J. ten Cate

Department of Cardiology, Thoraxcenter Erasmus

Medical Center, Rotterdam, The Netherlands

J. M. ten Berg

Department of Cardiology, St Antonius Hospital,

Nieuwegein, The Netherlands

J. W. R. Twisk

Department of Clinical Epidemiology and Biostatistics,

VU University Medical Center, Amsterdam,

The Netherlands blood flow. Methods Cine, contrast-enhanced (CE) imaging and breath-hold CMR phase contrast velocity mapping were performed at baseline and 1 and 6 months after ASA in seven patients. Changes of coronary blood flow were related to left ventricular (LV) mass reduction, enzyme release, volume of ethanol administered, LVOT gradient reduction, and LV rate pressure product (LVRPP). Results A significant mass reduction was observed both in the target septal myocardium and in the total myocardium (both $P<0.01$ ). Mean myocardial infarct size was $23 \pm 12 \mathrm{~g}$ (range 7.3-41.6 g). LVRPP decreased from $13,268 \pm 2,212$ to $10,685 \pm 3,918$ at 1 month $(P=0.05)$ and $9,483 \pm 2,496 \mathrm{mmHg}$ beats $/ \mathrm{min}$ at 6 months' follow-up $(P<0.01)$. LAD coronary blood flow decreased from $100 \pm 37 \mathrm{ml} / \mathrm{min}$ at baseline to $84 \pm 54 \mathrm{ml} / \mathrm{min}(P=0.09)$ at 1 month and $\quad 67 \pm 33 \mathrm{ml} / \mathrm{min}$ at 6 months follow-up $(P<0.01)$. A significant correlation was found between the change in LVRPP and LAD coronary flow at 1 month follow-up ( $r=0.83, P=0.02)$. CEinfarct size tended to modulate the blood flow changes over time $(P=0.12)$; no correlation was observed between enzyme release, volume of ethanol or both septal and total mass reduction and coronary blood flow. Conclusion The reduction in coronary blood flow is primarily associated with diminished LV loading conditions, whereas the induction of metabolically inactive myocardial scar tissue by ASA did not significantly influence the changes in coronary blood flow. 
Keywords Alcohol ablation .

Hypertrophic cardiomyopathy · Perfusion .

Magnetic resonance imaging

\section{Introduction}

Hypertrophic cardiomyopathy (HCM) is a primary cardiac disease characterized by hypertrophy of the myocardium in the absence of any systemic or other cardiac disease, which predominantly affects the interventricular septum [1]. In approximately one quarter of patients, the disease process of asymmetrical septal hypertrophy is complicated by left ventricular outflow tract (LVOT) obstruction due to bulging of the thickened septum into the outflow tract and abnormal anterior motion of the mitral valve during systole [2]. The inherent augmented loading conditions result in increased basal levels of oxygen utilization and coronary blood flow [3]. Coronary vasodilator reserve in HCM, however, is impaired due to coronary microvascular dysfunction. Consequently, metabolic demand frequently exceeds supply, leading to myocardial ischemia and its related symptoms $[4,5]$.

Alcohol septal ablation (ASA) has shown to successfully relieve LVOT obstruction with subsequent relief of symptoms in patients with hypertrophic obstructive cardiomyopathy (HOCM) $[6,7]$. This is achieved by ethanol infusion into septal branches of the left anterior descending coronary artery (LAD) resulting in an artificially induced septal myocardial infarction with regional myocardial wall thinning and widening of the LVOT [8]. The decrease of the pressure gradient leads to a reduction of the left ventricular (LV) end-diastolic pressure and LV wall stress [9]. Myocardial scarring and reduced metabolic demand caused by diminished loading conditions after ASA are expected to influence LAD blood flow [10]. Data to substantiate this hypothesis, however, are lacking.

Phase-contrast velocity measurements with cardiac magnetic resonance imaging (CMR) allows noninvasive quantification of coronary blood flow [11]. The present study was conducted to serially assess the impact of ASA on LAD resting blood flow using CMR phase-contrast imaging. Furthermore, the determinants of flow changes were explored.

\section{Methods}

Patients

Patients with HOCM scheduled to undergo ASA and eligible for CMR imaging were studied. The indication for ASA was based on a significant left ventricular outflow tract (LVOT) pressure gradient as documented by echocardiography and symptoms (NYHA functional class II-IV), despite medical treatment. Exclusion criteria were any absolute or relative contra-indication to CMR imaging (e.g., pacemaker, claustrophobia), atrial fibrillation, or failure to give informed consent. The septal ablation procedure has been described previously [8]. Results of the standard CMR protocol in a larger group have been published elsewhere $[8,12]$.

Patients underwent phase-contrast velocity measurements in addition to a standard CMR imaging protocol that included volume and mass measurements and delayed contrast-enhanced imaging prior to ASA and at 1 and 6 months after treatment. A total number of 16 patients were included in the study. Due to imaging artefacts and missing follow-up data, seven patients completed the study protocol and are reported in the current paper. One patient with recurrent symptoms underwent a redo procedure at 4 months after the initial ASA because no successful gradient reduction was achieved $(>50 \%)$.

The study protocol was approved by the Committee on Research Involving Human Subjects and the Medical Ethics Committee of the VU University Medical Center, Amsterdam.

\section{Echocardiography}

The LVOT pressure gradient was documented by Doppler echocardiography. In symptomatic patients a peak pressure gradient $\geq 50 \mathrm{mmHg}$ at rest was considered to be significant. One patient was symptomatic with a resting gradient $<50 \mathrm{mmHg}$. In this patient, provocation was applied using the Valsalva maneuver resulting in a pressure gradient during provocation of $\geq 50 \mathrm{mmHg}$.

CMR image acquisition

CMR was performed at baseline and 1 and 6 months after ASA on a 1.5 Tesla clinical scanner (Sonata, 
Siemens, Erlangen, Germany), using a four-element phased-array receiver coil. Cine- and delayed contrast-enhanced images were acquired with ECG gating and during repeated single breath-holds of 10-15 s depending on heart rate, coronary phase contrast velocity images were acquired using breath holds of 20-30 s. Cine images were acquired using a segmented steady-state free precession gradient-echo sequence in three long-axis views (2-, 3-, and 4chamber view) and in multiple short-axis views every $10 \mathrm{~mm}$, covering the entire left ventricle from base to apex.

Delayed contrast-enhanced (DCE) images were acquired 15-20 min after intravenous administration of $0.2 \mathrm{mmol} / \mathrm{kg}$ gadolinium-DTPA in the same views used in cine CMR, using a two-dimensional segmented inversion-recovery prepared gradient-echo sequence [13]. The DCE images were acquired to determine infarct size and location after the ablation procedure and to make sure that the infarct region was limited to the septum [8].

Coronal and transversal scout images were acquired to localize the left anterior descending (LAD) artery. Two-dimensional double oblique coronary angiographic sequence was obtained perpendicular to the direction of LAD flow with a breath-hold technique during inspiration. CMR phase contrast velocity measurements were performed in a plane perpendicular to the proximal LAD within one breath-hold (spatial resolution $1.2 \times 1.4 \times 5.5 \mathrm{~mm}^{3}$ ). A segmented $k$-space technique was used to obtain multiple phase-encoding steps (5) for each frame within the cardiac cycle, resulting in an acquisition window of $95 \mathrm{~ms}$. Other imaging parameters included a temporal resolution using echo sharing of $63 \mathrm{~ms}$, a flip angle of $25^{\circ}$, a field of view of $300 \times 244 \mathrm{~mm}^{2}$, an echo time of $3.6 \mathrm{~ms}$ and a scan duration of 20 heart beats. The encoding velocity was set between 40 and $125 \mathrm{~cm} / \mathrm{s}$. Heart rate and systemic arterial pressure were monitored during the imaging procedure.

CMR image analysis

\section{Analysis of cine images}

Global LV function parameters, including end-diastolic volume (EDV), end-systolic volume (ESV), ejection fraction (EF), total and septal myocardial mass were quantified using standard software (MASS version5, Medis, Leiden, the Netherlands). Endocardial and epicardial borders were traced manually in end-diastolic and end-systolic frames of all short-axis slices. Papillary muscles were included in the assessment of LV mass. The septum was defined as the myocardium between the anterior and posterior junctions of the right ventricle to the LV.

\section{Analysis of contrast-enhanced images}

Infarct size after ASA was measured by manual tracing of the hyperenhanced area within the septal myocardium with pixel signal intensity (SI) values $>$ SI of remote, non-enhanced myocardium +4 SD [8]. Central dark zones within the area of hyperenhancement were included.

\section{Analysis of left anterior descending blood flow}

Flow analysis was performed with the standard software (FLOW, Medis, Leiden, The Netherlands). The contour of the cross-sectioned LAD was visually determined on a magnitude image at mid diastole, the phase of the cardiac cycle in which the highest image quality was obtained. The area of the region of interest was kept constant over the cardiac cycle and repositioned at each time frame on the magnitude image. The averaged velocities were measured within each contour on the corresponding phase contrast image. The velocity of the myocardial motion was obtained by drawing a contour in the myocardial tissue close to the vessel of interest. To correct for cardiac motion the velocity within this contour was subtracted from the flow velocity within the vessel, resulting in a net forward velocity. The product of area and velocity yields instantaneous volumetric blood flow. Plots were made of phasic velocity and volume of blood flow versus time in the cardiac cycle (Fig. 1). Because a prospectively triggered electrocardiographic gating technique was used, no measurements could be obtained during the final $50 \mathrm{~ms}$ of the cardiac cycle. Data sets were interpolated over the whole cardiac cycle [11].

\section{Statistical analysis}

Results are expressed as mean \pm SD. Longitudinal data analysis was performed using generalized estimating equations (GEE) for serial measurements to 

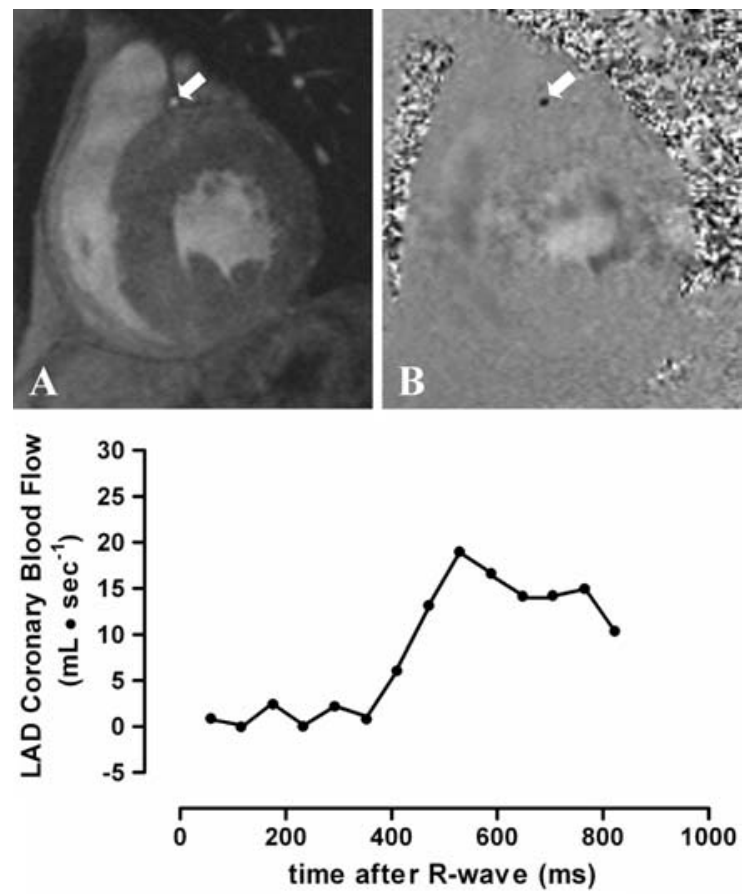

Fig. 1 Typical example of an anatomical (a) and corresponding velocity encoding CMR image (b) of the LAD coronary artery at mid-diastole (arrow). The graph represents the coronary blood flow curve of this patient

evaluate the changes in heart rate, systolic (SBP) and diastolic blood pressures (DBP), septal and total LV myocardial mass, LV volumes, LVOT pressure gradient (LVOTG), LV rate pressure product $[\mathrm{LVRPP}=(\mathrm{SBP}+\mathrm{LVOTG}) \times$ heart rate $]$ and LAD coronary artery blood volume flow from baseline to 1 and 6 months after ASA. In the GEEmodeling, time was added as a categorical variable indicated by dummies. Besides the crude development over time, the influence of certain modulating variables (e.g., amount of ethanol administered, cardiac enzyme release, infarct size measured by contrast-enhanced CMR, and serial change in the LVRPP) was investigated by adding the particular variables one at the time to the GEE-models in order to investigate whether the 'intercept' of the development was influenced. GEE analysis was also used to estimate the correlation between the changes in LAD blood volume flow and the changes in septal and total LV mass, LV volumes, ejection fraction, infarct size measured by contrast-enhanced CMR, and LVRPP. Linear regression analysis was used to analyze the relationship between LAD blood volume flow and the amount of ethanol administered, cardiac enzyme release and infarct size measured with contrastenhanced CMR. All GEE-analyses were performed with STATA (version 9); linear regression analyses were performed with SPSS version 14.0, and significance was set at a $P$-value $\leq 0.05$.

\section{Results}

Mean age was $46 \pm 12$ years (range 32-64, 5 males). The mean volume of ethanol injected during the ASA procedure was $2.9 \pm 1.2 \mathrm{ml}$ (range $1.5-5.0 \mathrm{ml}$ ). The mean peak CK and CK-MB release were 1,385 \pm $410 \mathrm{U}$ (range 807-1,997 U) and $207 \pm 54 \mathrm{U}$ (range 112-292 U), respectively. All patients were receiving one or more drugs (beta-blockers $[n=5]$, calcium channel blockers $[n=5]$, and antiarrhythmic drugs $[n=1]$ ), which were kept constant during the followup period.

At baseline, LVOT pressure gradient was $\geq 50 \mathrm{mmHg}$ in six patients $(87 \pm 11 \mathrm{mmHg}$; range $52-100 \mathrm{mmHg}$ ), and one had a provocable gradient (26 mmHg at rest, $80 \mathrm{mmHg}$ after Valsalva maneuver). A gradient reduction of more than $50 \%$ was achieved in six patients at a follow-up period of 6 months. All except one patient reported subjective improvement of exercise tolerance. The mean NYHA functional class improved significantly from $2.9 \pm 0.4$ to $1.8 \pm 0.9$ at 1 month and $1.3 \pm 0.7$ at 6 months after ASA $(P<0.01$ for trend).

Using delayed contrast-enhanced imaging after the procedure, septal infarct was demonstrated in all patients, none of the patients had evidence of infarctrelated hyperenhancement outside the interventricular septum. Mean myocardial infarct size was $23 \pm 12 \mathrm{~g}$ (range 7.3-41.6 g).

\section{Hemodynamic parameters}

Changes in hemodynamic parameters are summarized in Table 1. Compared to baseline, no change was observed in heart rate, systolic and diastolic arterial blood pressure at 1 and 6 months after ASA. The LVOT gradient decreased from $79 \pm 25$ to $34 \pm 48 \mathrm{mmHg}$ at 1 month to $23 \pm 35 \mathrm{mmHg}$ at 6 months after the procedure $(P<0.01)$. LVRPP decreased from $13,268 \pm 2,212$ to $10,685 \pm 3,918$ at 
Table 1 Basal hemodynamics and CMR derived parameters at baseline and after ASA

\begin{tabular}{|c|c|c|c|}
\hline & Baseline & 1 month follow-up & 6 months follow-up \\
\hline \multicolumn{4}{|l|}{ Hemodynamic parameters } \\
\hline Heart rate & $65 \pm 5$ & $65 \pm 6$ & $64 \pm 6$ \\
\hline Systolic blood pressure (mmHg) & $127 \pm 14$ & $130 \pm 14$ & $125 \pm 18$ \\
\hline Diastolic blood pressure (mmHg) & $74 \pm 13$ & $74 \pm 10$ & $76 \pm 9$ \\
\hline LV outflow gradient $(\mathrm{mmHg})$ & $79 \pm 25$ & $34 \pm 48 *$ & $23 \pm 35^{*}$ \\
\hline LVRPP $\left(* 10^{3} \mathrm{mmHg} \cdot\right.$ beats $\left./ \mathrm{min}\right)$ & $13,268 \pm 2,212$ & $10,685 \pm 3,918 \S$ & $9,483 \pm 2,496^{*}$ \\
\hline \multicolumn{4}{|l|}{ CMR derived parameters } \\
\hline LAD blood flow (ml/min) & $100 \pm 37$ & $84 \pm 54$ & $67 \pm 33 *$ \\
\hline LV end-diastolic volume (ml) & $164 \pm 46$ & $167 \pm 48$ & $177 \pm 37^{\S}$ \\
\hline LV end-systolic volume (ml) & $52 \pm 21$ & $54 \pm 18$ & $62 \pm 19^{\S}$ \\
\hline LV ejection fraction $(\%)$ & $69 \pm 7$ & $68 \pm 4$ & $66 \pm 5^{\S}$ \\
\hline Total LV mass (g) & $238 \pm 81$ & $220 \pm 79$ & $203 \pm 76^{*}$ \\
\hline Septal mass (g) & $77 \pm 25$ & $69 \pm 25$ & $64 \pm 23 *$ \\
\hline
\end{tabular}

Values expressed as mean $\pm \mathrm{SD}$

$L V$ Left ventricular; $R P P$ rate pressure product

$* P<0.01$ versus baseline, ${ }^{\S} P \leq 0.05$ versus baseline

1 month $(P=0.05)$ and $9,483 \pm 2,496 \mathrm{mmHg} \cdot$ beats/ min at 6 months follow-up $(P<0.01)$.

LV volumes, ejection fraction, regional mass

CMR derived values of $\mathrm{LV}$ volumes, EF, and regional myocardial mass are listed in Table 1. Ejection fraction decreased slightly, but significantly $(P<0.01)$, owing to a significant increase in LVESV $(P<0.05)$ over time after treatment. In addition, a significant mass reduction was observed both in the target septal myocardium and in the total myocardium (both $P<0.01$ ).

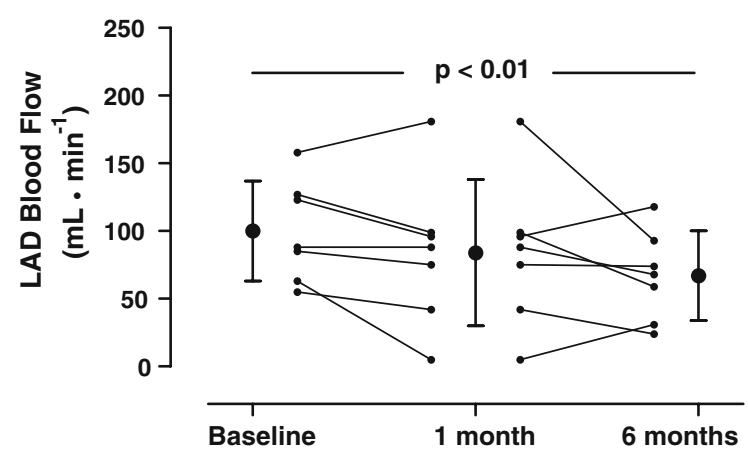

Fig. 2 Serial changes in LAD coronary blood volume flow at baseline and at 1 and 6 months after ASA, $P<0.01$ for trend
Correlation between changes in LAD blood flow and other parameters

LAD coronary blood flow decreased from $100 \pm 37 \mathrm{ml} / \mathrm{min}$ at baseline to $84 \pm 54 \mathrm{ml} / \mathrm{min}$ at 1 month $(P=0.09)$ and $67 \pm 33 \mathrm{ml} / \mathrm{min}$ at 6 months follow-up $(P<0.01$; Table 1; Fig. 2). The following modulators affected development over time: a larger

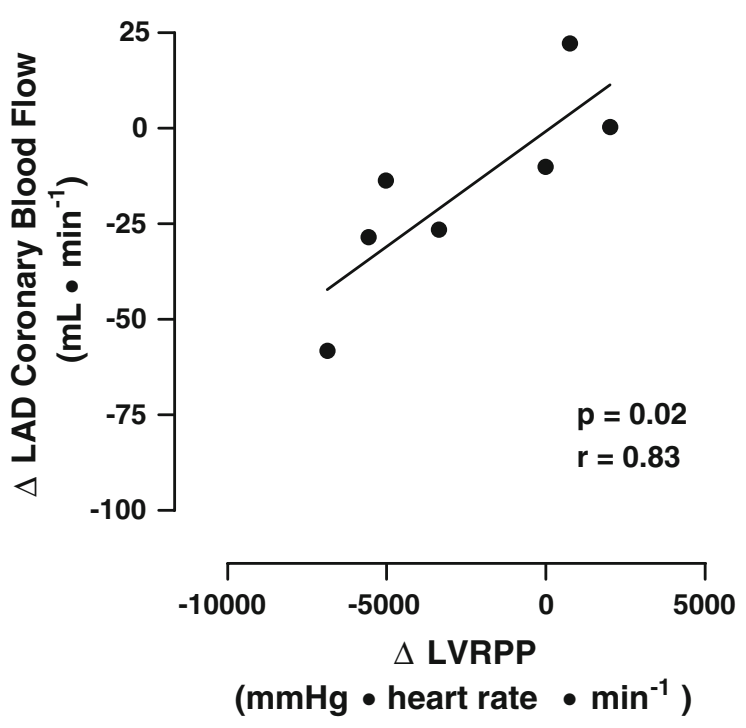

Fig. 3 Correlation between the changes in LVRPP and LAD coronary blood flow 
decrease of coronary blood flow was associated with a larger decrease of the LVOT pressure gradient ( $P=0.01$ for trend $)$ and LVRPP $(P<0.01$ for trend $)$. Figure 3 graphically displays the linear regression analysis between the change in LVRPP and LAD coronary flow at 1 month follow-up $(r=0.83$, $P=0.02$ ).

Coronary blood flow was not influenced by the amount of ethanol administered during the ASA procedure or cardiac enzyme release, although CEinfarct size tended to modulate the blood flow changes over time $(P=0.12)$. In addition, changes in myocardial mass did not exert influence on the observed flow changes.

\section{Discussion}

This is the first study that used CMR coronary flow quantification, using phase-contrast velocity measurements, to evaluate the effects of ASA on coronary blood flow in symptomatic patients with HOCM. Our data revealed a significant reduction of resting LAD coronary artery blood flow during the ensuing months of follow-up. We additionally explored the role of changes in hemodynamics and scar size induced by ablation therapy in relation to the observed changes in coronary blood flow over time.

Resting coronary blood flow is adjusted at the microvascular level in response to varying demand through autoregulation of the coronary microcirculation [14]. As heart rate and LV end-systolic cavity pressure are the major determinants of the energy requirement of the myocardium, these loading conditions primarily dictate the level of basal coronary blood flow [15]. The latter holds true under physiological as well as pathophysiological conditions, such as in patients with HCM [10]. In the present study, alcohol ablation significantly reduced the hemodynamic loading conditions, i.e., LVRPP. These changes were solely determined by the reduction in LVOT pressure gradient as heart rate and arterial blood pressure were unaffected by the procedure. As hypothesized, the changes in coronary blood flow were directly related to these alterations in loading conditions. These observations are in line with Cannon et al. [16] who elegantly demonstrated a reduction of great cardiac vein flow as well as global myocardial oxidative metabolism 6 months after surgical myectomy in symptomatic HOCM patients. Similar to the present findings, these changes were directly governed by the magnitude of outflow tract pressure gradient relief. More recently, using cardiac positron emission tomography, Jörg-Ciopor et al. [17] revealed a lower perfusion per grams of septal tissue after surgical myectomy compared with medically treated patients. Moreover, Rajappan et al. [18] have shown that these physiological flow changes can also be observed after gradient reduction through valve replacement in patients with aortic valve stenosis.

The fundamental approach of alcohol ablation is based on artificially inducing a fairly large myocardial infarct in the hypertrophied myocardial septum [8]. The procedure itself results in obliteration of a septal branch of the LAD [6]. Furthermore, the generated scar is metabolically inactive relative to the previous myocardium subtended by the sacrificed coronary artery [19]. As the current flow measurements were presumably performed proximal to the ablation site of the LAD, just distal to the junction of the left main coronary artery and circumflex, these conditions in theory are expected to influence coronary blood flow. Nonetheless, a clear relationship between CE-estimated infarct size and changes in coronary blood flow could not be established, although a trend was observed $(P=0.12)$. It therefore appears, that the reduction in coronary blood flow is primarily instigated by altered LV loading conditions as opposed to the induction of metabolically inactive myocardial scar tissue. The lack of impact of scar on coronary blood flow is not well understood and requires further investigation.

Regardless of the underlying mechanisms that are responsible for the observed decrease in coronary blood flow, the observation is important from a pathophysiological point of view. The occurrence of coronary microvascular dysfunction in $\mathrm{HCM}$ has been firmly established and shown to be of important prognostic relevance [20]. The reduction in loading conditions by ASA and the concomitant reduction in basal coronary flow could result in enhanced coronary flow reserve. In other words, relief of resting loading conditions may result in less frequent episodes of ischemia for a given level of exertion, and could explain the reported reduction for anginal symptoms after treatment, and may ultimately contribute to the suggested beneficial effects on prognosis [6, 21]. Unfortunately, hyperemic LAD 
flow was not determined in the present study and therefore the postulated enhancement of flow reserve remains to be investigated. Preliminary data from Soliman et al. [22], however, does suggest an increase in flow reserve after septal alcohol ablation.

\section{Limitations}

Only a limited number of patients with HOCM were studied, and the results should be interpreted with care. Nevertheless, the coronary flow data convincingly indicate significant changes in coronary blood flow in the LAD coronary artery after ASA.

The single breath-hold phase contrast velocity quantification technique used in this study has important limitations. First, it requires a long breath-hold (20-30 s), which is difficult to obtain in every patient. Second, the position of the breath-holding may vary, which can result in a different image position from the originally planned. Third, the technique has a large acquisition window which results in some image blurring because of cardiac motion [23]. Newer techniques such as the use of spiral imaging reduced this acquisition window and will result in a higher accuracy [24], but this could not be achieved on the system used. The slice thickness $(5.5 \mathrm{~mm})$ is not likely to importantly affect the through plane velocity measurement, because the imaged plane was positioned perpendicular to a relative straight segment of the proximal left anterior descending coronary artery. Fourth, the in-plane spatial resolution of the CMR acquisition is limited with respect to the vessel diameter, which is expected to cause variability on the cross-sectional area assessment, and thereby on the volume flow. These methodological issues prohibit its use in routine clinical practice and results in unsuccessful measurements in a substantial number of patients. Clearly, this technique is not ready for clinical use but can be useful for research purposes, as presented in the current manuscript. Moreover, the same methodology was used before and after septal ablation. Attention was given to position the CMR slice-position in an identical position within the left anterior descending coronary artery. Thus, by using each patient as his own control, errors in methodology are expected to remain constant and thus allow valid observation of the impact of the ablation procedure on coronary artery flow.
In conclusion, we demonstrated that the reduction of the LVOT pressure gradient by alcohol septal ablation in symptomatic patients with HOCM was accompanied by a significant decrease in left anterior descending coronary resting blood flow. No significant correlation was found between the artificially induced septal myocardial infarct size and the reduction in coronary blood flow over time. These findings support the hypothesis that diminished loading conditions after successful relief of the LVOT pressure gradient decreases the oxygen utilization and consequently, coronary blood flow after ASA.

Acknowledgments This study was supported by grant 99.203 from the Netherlands Heart Foundation and the Interuniversity Cardiology Institute of the Netherlands.

\section{References}

1. Maron BJ (2002) Hypertrophic cardiomyopathy: a systematic review. JAMA 287:1308-1320. doi:10.1001/jama. 287.10.1308

2. Maron MS, Olivotto I, Betocchi S et al (2003) Effect of left ventricular outflow tract obstruction on clinical outcome in hypertrophic cardiomyopathy. N Engl J Med 348:295-303. doi:10.1056/NEJMoa021332

3. Cannon ROIII, Schenke WH, Maron BJ et al (1987) Differences in coronary flow and myocardial metabolism at rest and during pacing between patients with obstructive and patients with nonobstructive hypertrophic cardiomyopathy. J Am Coll Cardiol 10:53-62

4. Camici PG, Crea F (2007) Coronary microvascular dysfunction. N Engl J Med 356:830-840. doi:10.1056/NEJMra 061889

5. Cannon ROIII, Rosing DR, Maron BJ et al (1985) Myocardial ischemia in patients with hypertrophic cardiomyopathy: contribution of inadequate vasodilator reserve and elevated left ventricular filling pressures. Circulation 71:234-243

6. Sigwart U (1995) Non-surgical myocardial reduction for hypertrophic obstructive cardiomyopathy. Lancet 346:211-214. doi:10.1016/S0140-6736(95)91267-3

7. Lakkis NM, Nagueh SF, Dunn JK, Killip D, Spencer WHIII (2000) Nonsurgical septal reduction therapy for hypertrophic obstructive cardiomyopathy: one-year follow-up. J Am Coll Cardiol 36:852-855. doi:10.1016/S0735-1097(00) 00767-1

8. van Dockum WG, ten Cate FJ, ten Berg JM et al (2004) Myocardial infarction after percutaneous transluminal septal myocardial ablation in hypertrophic obstructive cardiomyopathy: evaluation by contrast-enhanced magnetic resonance imaging. J Am Coll Cardiol 43:27-34. doi: 10.1016/j.jacc.2003.08.031

9. Roberts R, Sigwart U (2005) Current concepts of the pathogenesis and treatment of hypertrophic cardiomyopathy. Circulation 112:293-296. doi:10.1161/01.CIR.0000 146788.30724.0A 
10. Knaapen P, Germans T, Camici PG et al (2008) Determinants of coronary microvascular dysfunction in symptomatic hypertrophic cardiomyopathy. Am J Physiol Heart Circ Physiol 294:H986-H993. doi:10.1152/ajpheart.00233.2007

11. Hundley WG, Lange RA, Clarke GD et al (1996) Assessment of coronary arterial flow and flow reserve in humans with magnetic resonance imaging. Circulation 93:15021508

12. van Dockum WG, Beek AM, ten Cate FJ et al (2005) Early onset and progression of left ventricular remodeling after alcohol septal ablation in hypertrophic obstructive cardiomyopathy. Circulation 111:2503-2508. doi:10.1161/01. CIR.0000165084.28065.01

13. Wu E, Judd RM, Vargas JD, Klocke FJ, Bonow RO, Kim RJ (2001) Visualisation of presence, location, and transmural extent of healed Q-wave and non-Q-wave myocardial infarction. Lancet 357:21-28. doi:10.1016/ S0140-6736(00)03567-4

14. Westerhof N, Boer C, Lamberts RR, Sipkema P (2006) Cross-talk between cardiac muscle and coronary vasculature. Physiol Rev 86:1263-1308. doi:10.1152/physrev. 00029.2005

15. Braunwald E (1971) Control of myocardial oxygen consumption: physiologic and clinical considerations. Am J Cardiol 27:416-432. doi:10.1016/0002-9149(71)90439-5

16. Cannon RO III, McIntosh CL, Schenke WH, Maron BJ, Bonow RO, Epstein SE (1989) Effect of surgical reduction of left ventricular outflow obstruction on hemodynamics, coronary flow, and myocardial metabolism in hypertrophic cardiomyopathy. Circulation 79:766-775

17. Jorg-Ciopor M, Namdar M, Turina J et al (2004) Regional myocardial ischemia in hypertrophic cardiomyopathy: impact of myectomy. J Thorac Cardiovasc Surg 128:163169. doi:10.1016/j.jtcvs.2003.11.003

18. Rajappan K, Rimoldi OE, Camici PG, Bellenger NG, Pennell DJ, Sheridan DJ (2003) Functional changes in coronary microcirculation after valve replacement in patients with aortic stenosis. Circulation 107:3170-3175

19. Kuhn H, Gietzen FH, Schafers M et al (1999) Changes in the left ventricular outflow tract after transcoronary ablation of septal hypertrophy (TASH) for hypertrophic obstructive cardiomyopathy as assessed by transoesophageal echocardiography and by measuring myocardial glucose utilization and perfusion. Eur Heart J 20:18081817. doi:10.1053/euhj.1999.1692

20. Cecchi F, Olivotto I, Gistri R, Lorenzoni R, Chiriatti G, Camici PG (2003) Coronary microvascular dysfunction and prognosis in hypertrophic cardiomyopathy. N Engl J Med 349:1027-1035. doi:10.1056/NEJMoa025050

21. Maron MS, Olivotto I, Betocchi S et al (2003) Effect of left ventricular outflow tract obstruction on clinical outcome in hypertrophic cardiomyopathy. N Engl J Med 348:295-303. doi:10.1056/NEJMoa021332

22. Soliman OI, Geleijnse ML, Michels M, Dijkmans PA, Nemes A, van Dalen BM, Vletter WB, Serruys PW, ten Cate FJ (2008) Effect of successful alcohol septal ablation on microvascular function in patients with obstructive hypertrophic cardiomyopathy. Am J Cardiol 101(9):13211327. doi:10.1016/j.amjcard.2007.12.032

23. Hofman MB, Wickline SA, Lorenz CH (1998) Quantification of in-plane motion of the coronary arteries during the cardiac cycle: implications for acquisition window duration for MR flow quantification. J Magn Reson Imaging 8:568-576. doi:10.1002/jmri.1880080309

24. Keegan J, Gatehouse PD, Mohiaddin RH, Yang GZ, Firmin DN (2004) Comparison of spiral and FLASH phase velocity mapping, with and without breath-holding, for the assessment of left and right coronary artery blood flow velocity. J Magn Reson Imaging 19:40-49. doi:10.1002/ jmri.10434 